INPLASY

PROTOCOL

To cite: Song et al. Effects of Aromatherapy on Sleep

Disorders: A protocol for systematic review and metaanalysis. Inplasy protocol 202140035. doi:

10.37766/inplasy2021.4.0035

Received: 07 April 2021

Published: 07 April 2021

Corresponding author:

Lisheng Jiang

502878691@qq.com

Author Affiliation: Institute of Health Preservation,Jiangxi University of Traditional Chinese

Medicine

Support: None.

Review Stage at time of this submission: Preliminary searches.

Conflicts of interest: None declared.

\section{Effects of Aromatherapy on Sleep Disorders: A protocol for systematic review and meta-analysis}

Song, X1; Peng, J2; Jiang, W3; Ye, M4; Jiang, L55.

Review question / Objective: The purpose of this study is to systematically evaluate the effectiveness of aromatherapy in improving sleep quality in people with sleep disorders.

Condition being studied: Sleep disorders are a serious health and public health problem in today's society, mainly manifested as insomnia, sleep-related breathing disorders, excessive sleepiness and parasomnias, etc, which seriously affects people's daily life, work and physical and mental health, and is closely related to cardiovascular and cerebrovascular diseases, malignant tumors, and psychological diseases. According to clinical statistics, about $10 \%$ to $20 \%$ of adolescents and adults suffer from insomnia, and the incidence of sleep disorders in the elderly is $30 \%$ to $40 \%$. Nearly $90 \%$ of the elderly experience sleep problems such as difficulty falling asleep, waking up early or increasing the number of awakenings, etc. Although insomnia is not a critical illness, long-term insomnia not only seriously affects people's work and quality of life, it is also an important risk factor for mental and psychological diseases, cardiovascular diseases, and diabetes, which increases the medical burden of society. Therefore, it is necessary to actively pay attention to sleep problems and seek practical and effective treatments. Aromatherapy is a kind of essential oil therapy that directly uses plant spices or indirectly extracts essence from spices. It was first used in the beauty industry and then gradually expanded to the medical field. It is mainly used to prevent, relieve or treat certain diseases.

INPLASY registration number: This protocol was registered with the International Platform of Registered Systematic Review and Meta-Analysis Protocols (INPLASY) on 07 April 2021 and was last updated on 07 April 2021 (registration number INPLASY202140035).

\section{INTRODUCTION}

Review question / Objective: The purpose of this study is to systematically evaluate the effectiveness of aromatherapy in improving sleep quality in people with sleep disorders. 
Condition being studied: Sleep disorders are a serious health and public health problem in today's society, mainly manifested as insomnia, sleep-related breathing disorders, excessive sleepiness and parasomnias, etc, which seriously affects people's daily life, work and physical and mental health, and is closely related to cardiovascular and cerebrovascular diseases, malignant tumors, and psychological diseases. According to clinical statistics, about $10 \%$ to $20 \%$ of adolescents and adults suffer from insomnia, and the incidence of sleep disorders in the elderly is $30 \%$ to $40 \%$. Nearly $90 \%$ of the elderly experience sleep problems such as difficulty falling asleep, waking up early or increasing the number of awakenings, etc. Although insomnia is not a critical illness, long-term insomnia not only seriously affects people's work and quality of life, it is also an important risk factor for mental and psychological diseases, cardiovascular diseases, and diabetes, which increases the medical burden of society. Therefore, it is necessary to actively pay attention to sleep problems and seek practical and effective treatments. Aromatherapy is a kind of essential oil therapy that directly uses plant spices or indirectly extracts essence from spices. It was first used in the beauty industry and then gradually expanded to the medical field. It is mainly used to prevent, relieve or treat certain diseases.

\section{METHODS}

Participant or population: Patients with sleep disorders, sleep disorders assessed by tools (PSQI, SRSS, ICSD, etc.), age $\geq 18$ years old, there are no limits to research subjects age, gender, race, condition duration, or intensity. Participants with serious underlying diseases, pregnant or lactating women, will be excluded.

Intervention: The experimental group only use aromatherapy.

Comparator: The control group given routine care, placebo, blank control.
Study designs to be included: The type of literature research is RCTs.

Eligibility criteria: (1) The type of literature research is RCT. (2) Study participants: Patients with sleep disorders, sleep disorders assessed by tools (PSQI, SRSS, ICSD, etc.), age $\geq 18$ years old, there are no limits to research subjects age, gender, race, condition duration, or intensity. Participants with serious underlying diseases, pregnant or lactating women, will be excluded. (3)Experimental group: only use aromatherapy; control group: given routine care, placebo, blank control.(4) Outcome indicators include PSQI (Pittsburgh sleep quality index),SRSS Scale,Clinical effectiveness, and adverse reactions, including at least one of them. (5) There was no statistical difference in the baseline comparison of diabetic foot patients.

Information sources: Computer search database: CNKI, SinoMed, Wanfang, VIP, PubMed, Web of science, EMbase. The search time range is from the establishment of the database to April 5, 2021. Use a combination of subject terms and free words to search.In order to avoid omissions, search scope for including subject words, keywords, or full text.Search terms are: "Aromatherapy," "Aromatic therapy," "Aroma," "Aromatic," "Fragrance," "Aromatic substances," "Essential oil," "Sleep," "Insomnia," "Sleep disorder," "Sleep quality," "Comorbid insomnia," "Randomized controlled trial," "RCT".

Main outcome(s): (1)PSQI (Pittsburgh sleep quality index): PSQI is used to assess the sleep quality of subjects in the last month. It consists of 19 self-evaluated items and 5 other-evaluated items, of which the 19th self-evaluated item and 5 other-evaluated items do not participate in scoring. 18 items constitute 7 components, and each component is scored on a scale of 0 to 3 . The cumulative score of each component is the PSQI total score, the total score range is $0 \sim 21$, the higher the score, the worse the sleep quality. (2)SRSS Scale: There are 10 
items in SRSS, and each item is divided into 5 grades (1 5). The higher the score, the more serious the sleep problem. The lowest score of this scale is $\mathbf{1 0}$ (basically no sleep problems), and the highest score is 50 (most serious). (3) Clinical effectiveness including 4 grades of recovery, obvious effect, effective and ineffective. Effective rate $=[($ recovery + obvious effect + effective)/total number of cases] $\times 100 \%$. (4)Adverse reactions.

Quality assessment / Risk of bias analysis: Two researchers independently evaluated the risk of bias based on the RCT bias risk assessment tool recommended by the Cochrane Handbook, and cross-checked the results. If there is any disagreement, it will be resolved through discussion or negotiation with the third researcher.

Strategy of data synthesis: RevMan5.3 software was used for Meta analysis. Mean difference (MD) was used for measurement data, odds ratio (OR) was used for classification data, and $95 \%$ confidence interval $(95 \% \mathrm{Cl})$ was used as the statistical analysis quantity. The heterogeneity among the included research results was analyzed by chi-square test (test level $a=0.1$ ), and the degree of heterogeneity was quantitatively judged by combining with $\mathbf{1 2}$. If there is no statistical heterogeneity ( $P>1,12<50 \%)$ between the results of each study, the fixed effects model is used; if there is statistical heterogeneity (P50\%) between the results of each study, then subgroup analysis is used. For the multi-arm experiment included in this meta-analysis, grouping and merging are adopted, the multi-arm experiment is converted to a two-arm experiment, and the mean standard deviation is combined as required.

Subgroup analysis: Subgroup analysis can try to classify and compare the sources of different intervention objects to determine whether the patient chooses the following as the analysis elements in the subgroup analysis because the treatment effect is significant under what circumstances.
Sensitivity analysis: The purpose of sensitivity analysis is to reduce the heterogeneity of research. When the heterogeneity is high, sensitivity analysis should be carried out to exclude the research progress involved one by one, observe the changes in the heterogeneity results, and make the experimental results more reliable and stable.

Country(ies) involved: China.

Keywords: Aromatherapy, Sleep Disorders, Protocol, Systematic review.

Contributions of each author:

Author 1 - Xin Song.

Author 2 - Jiahua Peng.

Author 3 - Weiyu Jiang.

Author 4 - Minghua Ye.

Author 5 - Lisheng Jiang. 